Rapid Reviews COVID-19•

\title{
Review 1: "Small-molecule ligands can inhibit -1 programmed ribosomal frameshifting in a broad spectrum of coronaviruses"
}

Ewan Plant ${ }^{1}$

1"US FDA CBER UNITED STATES

Published on: Dec 05, 2021

License: Creative Commons Attribution 4.0 International License (CC-BY 4.0). 


\section{RR:C19 Evidence Scale rating by reviewer:}

- Reliable. The main study claims are generally justified by its methods and data. The results and conclusions are likely to be similar to the hypothetical ideal study. There are some minor caveats or limitations, but they would/do not change the major claims of the study. The study provides sufficient strength of evidence on its own that its main claims should be considered actionable, with some room for future revision.

$* * * * * * * * * * * * * * * * * * * * * * * * * * * * * * * * * * * * * * *$

\section{Review:}

The article by Munshi et al. describes the identification and analysis of small molecules that are able to inhibit a coronavirus regulatory element in an in vitro assay. Coronaviruses use an RNA structural element called an RNA pseudoknot to regulate the expression of viral RNA and viral proteins. Inhibiting this control point disrupts the virus lifecycle. Munshi et al. screened a library of FDA-approved drugs for their ability to modulate the regulatory element. Four molecules that inhibited the signal were selected for additional analysis. These were compared with two molecules previously found to inhibit the regulatory element. One of the molecules identified inhibited a number of coronavirus RNA pseudoknots but not the regulatory signals from two unrelated viruses-demonstrating potential as a therapeutic for coronavirus infection.

The -1 programmed ribosomal frameshifting mechanism has been identified in all coronaviruses. This regulatory feature includes a three-stem RNA pseudoknot and disruption of this has been shown to abrogate virus replication. There is variation in the RNA pseudoknot among different coronaviruses. Munshi et al. use the available genomic information to find the variability in bat coronaviruses. Six different frameshift signals were used in the study. The value of this is that the drug screen identifies molecules that act on the range of coronavirus regulatory elements, including those from strains that are not part of the current pandemic. This also reduces the risk that a molecule loses efficacy if the coronavirus evolves.

The authors used a panel of bat coronaviruses to create the three-stemmed pseudoknot containing constructs for the assays. A two-stem pseudoknot and stemloop structure from a plant virus and HIV were also used in the assay. The molecules identified had the greatest impact on the three-stemmed pseudoknots indicating specificity for that type. 
Modulation of frameshift signals has been shown to significantly attenuate the growth of viruses including coronaviruses. These studies are usually performed in tissue culture. The impact these molecules have on coronavirus replication needs to be evaluated.

The authors focus on the impact the molecules have on frameshifting. Based on the data presented, it is easy to assume that the mechanism of action is by disrupting the frameshifting mechanism, but it is also possible that the molecule inhibits coronaviruses via an off-target mechanism such as preventing virus entry. The most promising molecule, nafamostat, has been already been used for COVID-19 treatment (https://doi.org/10.1016/j.amsu.2021.102560) and the authors should comment on this. 